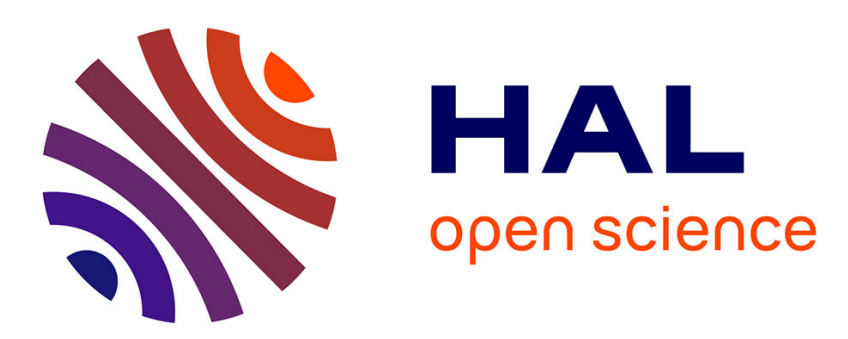

\title{
Champ diffusé par un tube élastique: comparaison entre la théorie géométrique de la diffraction et le formalisme modal
}

\author{
J.-L. Rousselot, P. Millerat
}

\section{- To cite this version:}

J.-L. Rousselot, P. Millerat. Champ diffusé par un tube élastique: comparaison entre la théorie géométrique de la diffraction et le formalisme modal. Journal de Physique IV Proceedings, 1994, 04 (C5), pp.C5-853-C5-856. 10.1051/jp4:19945185 . jpa-00252867

\section{HAL Id: jpa-00252867 https://hal.science/jpa-00252867}

Submitted on 1 Jan 1994

HAL is a multi-disciplinary open access archive for the deposit and dissemination of scientific research documents, whether they are published or not. The documents may come from teaching and research institutions in France or abroad, or from public or private research centers.
L'archive ouverte pluridisciplinaire HAL, est destinée au dépôt et à la diffusion de documents scientifiques de niveau recherche, publiés ou non, émanant des établissements d'enseignement et de recherche français ou étrangers, des laboratoires publics ou privés. 


\title{
Champ diffusé par un tube élastique : comparaison entre la théorie géométrique de la diffraction et le formalisme modal
}

\section{J.-L. ROUSSELOT et P. MILLERAT}

Le Centre Thomson d'Applications Radars, 6 rue Nieuport, BP. 86, 78143 Velizy-Villacoublay cedex, France

\begin{abstract}
Résumé : On analyse et on évalue chacune des contributions à la pression diffusée par un tube cylindrique immergé insoné par une onde acoustique plane. Ces contributions ont pour origine le rayonnement des ondes circonférentielles d'une part et la pression résultant des rayons réfractés et multiplement réfléchis d'autre part. Le résultat de la superposition de ces contributions obtenues par l'application de la Théorie Géométrique de la Diffraction est confronté aux valeurs prédites par l'approche modale classique.
\end{abstract}

Abstract : A numerical analysis is performed on each contribution for the pressure field radiated by an immersed cylindrical shell which are the circumferential waves and the geometrical waves (reflected and transmitted) using the Geometrical Theory of Diffraction. By adding all the contributions, the result is compared with those obtained by a modal approach.

\section{INTRODUCTION.}

L'étude du champ diffusé par un tube cylindrique en incidence normale a été entreprise depuis de nombreuses années. Il en existe une solution exacte dont l'expression est donnée par une série convergente appelée série modale. Pour un point d'observation de coordonnées $(r, \varphi)$, le champ diffusé s'exprime par :

$$
p_{d}=P_{o} e^{-i \omega t} \sum_{n=0}^{\infty} \varepsilon_{n} i^{n} \frac{D_{n}^{[1]}\left(x_{1}\right)}{D_{n}\left(x_{1}\right)} H_{n}^{(1)}\left(k_{1} r\right) \cos (n \varphi)
$$

Dans cette relation, $P_{0}$ désigne un facteur de normalisation pour le champ incident, $H_{n}{ }^{(1)}\left(k_{1} r\right)$ est la fontion de Hankel d'argument $k_{1} r$ où $k_{1}$ est le nombre d'onde du signal harmonique de pulsation $\omega$ dans le fluide externe, $\mathrm{D}_{\mathrm{n}}{ }^{[1]}\left(\mathrm{x}_{1}\right)$ et $\mathrm{D}_{\mathrm{n}}\left(\mathrm{x}_{1}\right)$ sont deux déterminants obtenus à partir des conditions de continuité des déplacements et des contraintes au niveau des interfaces. On a noté $\mathrm{x}_{1}=\mathrm{k}_{\mathrm{1}}$ a où a est le rayon externe du tube $\left(\varepsilon_{0}=1, \varepsilon_{\mathrm{n}}=2, \mathrm{n} \neq 0\right.$ ). Le nombre de termes utiles est de l'ordre de grandeur du paramètre $\mathrm{x}_{1}$. Pour les grandes valeurs de $\mathrm{x}_{1}$, la convergence est lente et la sommation d'un nombre élevé de termes peut conduire à des résultats numériques imprécis. De plus, il est assez difficile de donner une interprétation physique simple des mécanismes de diffusion par cette approche. Pour ces deux raisons, une nouvelle approche fondée sur la Transformation de Sommerfeld-Watson a été développée [1]. 
La mise en oeuvre de cette approche consiste à superposer les contributions des ondes de surface et des rayons géométriques réfléchis et transmis.

\section{CONTRIBUTIONS DES ONDES DE SURFACE.}

Le formalisme conduisant à l'évaluation de la contribution des ondes de surface est développé dans la référence [1] et a été appliqué au cylindre fluide [2]. Il s'applique également dans le cas du tube. Soit $\nu_{1}$ l'extension complexe de l'indice $\mathrm{n}$ de la relation (1). On désigne par $\nu_{1}$ une racine de l'équation $\mathrm{D}\left(\nu, \mathrm{x}_{1}\right)=0$. A cette racine est associée une onde de surface dont les caractéristiques sont liées à la valeur de la partie réelle $\operatorname{Re}\left(\nu_{1}\right)$ et de la partie imaginaire $\operatorname{Im}\left(\nu_{1}\right)$ de cette racine. A partir de ces valeurs, on peut distinguer deux familles d'ondes : les ondes rampantes de type Franz notées F1, F2 et F3, ... de forte atténuation. On range également dans cette famille l'onde de Stoneley (notée $1=0$ ) d'atténuation plus faible. L'autre famille est constituée des ondes de surface élastique (onde de Rayleigh, $1=1$ et les ondes de Lamb, $1=2,3, \ldots)$. La valeur de la pression rayonnée par chacune de ces ondes au point d'observation a été calculée et tracée sur la figure 1 en fonction de l'angle $\varphi$. On constate que chaque courbe présente une discontinuité pour un certain angle $\varphi_{\mathrm{I}}^{(\text {() }}$ différent pour chaque onde. Soit $\alpha_{\mathrm{c}}{ }^{(\mathrm{I})} 1$ 'angle d'excitation, son expression peut s'écrire :

$$
\alpha_{c}^{(l)}=\sin ^{-1}\left\{\frac{\operatorname{Re}\left(v_{l}\right)}{x_{1}}\right\}
$$

et l'angle $\varphi_{1}^{(l)}$ s'exprime par la relation :

$$
\varphi_{I}^{(n)}=\pi-2 \alpha_{c}^{(n)}+\sin ^{-1}\left\{\frac{a}{r} \sin \alpha_{c}^{(l)}\right\},
$$

Pour les ondes rampantes, le calcul de la relation (3) donne $\varphi_{\mathrm{I}}^{(\mathrm{l})}=11,54^{\circ}$ et pour chacune des ondes de surface élastique $\varphi_{\mathrm{I}}^{(\mathrm{l})}=122^{\circ}$ pour $1=1, \varphi_{\mathrm{I}}^{(\mathrm{l})}=141^{\circ}$ pour $1=2$ et $\varphi_{\mathrm{I}}^{(1)}=159^{\circ}$ pour $1=3$. Par ailleurs, la modulation visible sur les courbes $s^{\prime}$ interprète en considérant qu'il existe en fait pour un même indice 1 deux ondes circulant en sens inverse 1'une de l'autre. Ces ondes sont en phase pour $\varphi=0$ et $\varphi=180^{\circ}$, valeurs pour lesquelles leurs trajets sont égaux.

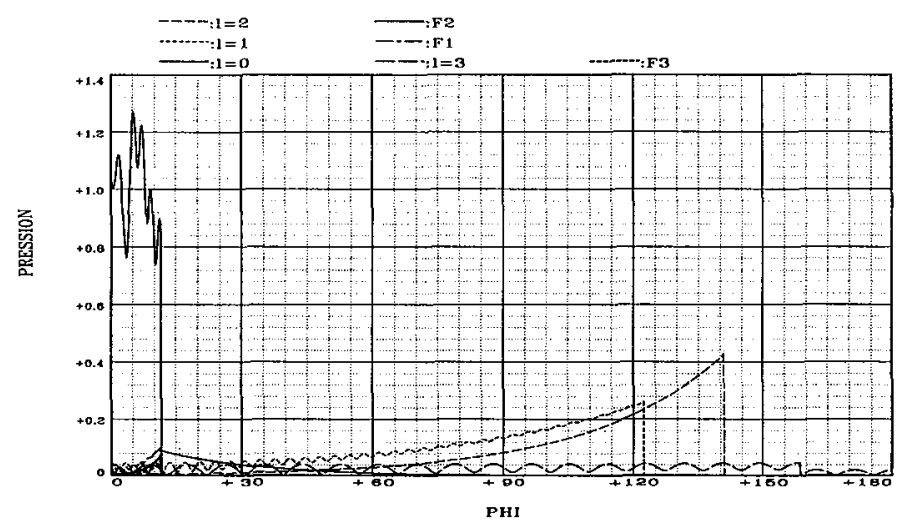

Fig. 1 : Pression rayonnée par les ondes de surface au point d'observation $\mathrm{x}_{1}=100, \mathrm{r} / \mathrm{a}=5, \mathrm{~b} / \mathrm{a}=0.90, \mathrm{~b}=$ rayon interne 


\section{RAYONS GEOMETRIQUES.}

La prise en compte de ces rayons est fondée également sur la théorie de Sommerfeld-Watson [1] et l'application présentée ici s'inspire des travaux [3]. Un rayon incident au point d'impact A1 (fig. 2) formant un angle $\alpha$ avec la normale en ce point est réfracté dans le milieu interne suivant un rayon à polarisation longitudinale (noté $\mathrm{L}$ ) et un rayon à polarisation transversale (noté $\mathrm{T}$ ). Ces deux rayons sont réfléchis sur l'interface interne, puis sur l'interface externe et ainsi de suite jusqu'au point d'émergence $A_{n+1}$ où $n$ est le nombre de trajets élémentaires. Pour $n$ trajets, on considère $m$ trajets $(T)$ (on $a(n-m)$ trajets $\mathrm{L})$. Pour chaque chemin $(\mathrm{m}, \mathrm{n})$ on fait correspondre un coefficient de divergence géométrique $\mathrm{D}_{\mathrm{n}, \mathrm{m}}(\alpha)$, obtenu par des considérations sur la conservation de l'énergie, et un coefficient d'interaction $\mathrm{G}_{\mathrm{n}, \mathrm{m}}(\alpha)$ obtenu à partir des coefficients de réflexion et de transmission. Dans la présente application, on a utilisé les coefficients de la lame plane [4]. L'expression du champ produit par tous les rayons géométriques s'écrit alors :

$$
p_{g e ́}=P_{o} e^{i \frac{c_{i}}{a} x_{1}} \sum_{n=0}^{\infty} \sum_{m=0}^{n} \frac{G_{n, m}(\alpha)}{\sqrt{D_{m m}(\alpha)}} e^{-i \frac{x_{1} c_{1}}{a} t(O P)}
$$

Ayant établi pour tous les cas possibles des relations exprimant l'angle $\alpha$ en fonction de l'angle $\varphi$, il est possible de calculer les valeurs des coefficients $G_{n, m}(\alpha), D_{n, m}(\alpha)$ et également le temps de parcours de chaque rayon $(n, m)$.

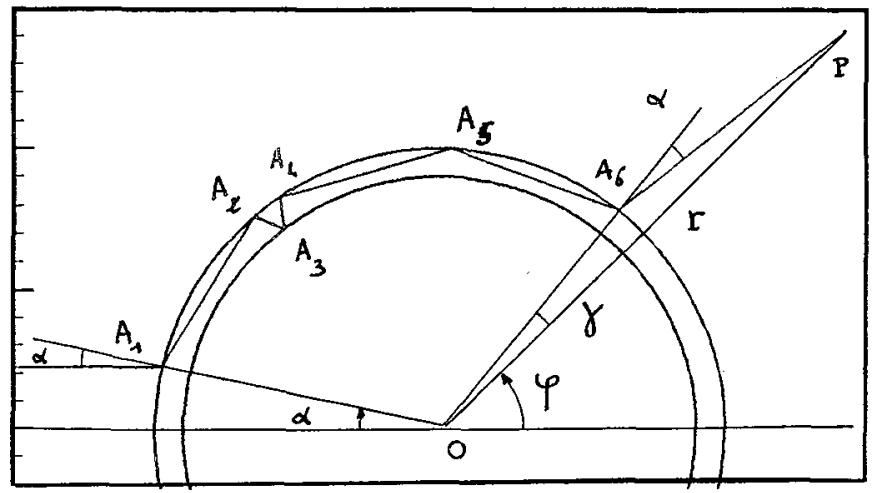

Fig. 2 : Construction géométrique des rayons réfléchis et réfractés pour $\mathrm{n}=5, \mathrm{~m}=2$

Numériquement, la série (4) converge lentement puisque le nombre de trajets n doit être supérieur à $n=16$ pour que l'erreur commise soit inférieure à $10 \%$. En sommant en module et en phase la pression rayonnée par chacune des ondes de surface et la pression $\mathrm{p}_{\mathrm{gé}}$, on obtient la courbe de la figure 3 tracée sur le même graphique que la courbe donnée par la série modale. L'accord est en général assez bon. Toutefois, dans l'intervalle $110<\varphi<155$ des différences sensibles entre les deux courbes montrent que les transitions ombre-lumière ne sont pas suffisamment bien restituées. Ce point est en cours d'analyse. Pour les applications numérique le tube est en aluminimum avec $\mathrm{c}_{\mathrm{L}}=6420, \mathrm{c}_{\mathrm{T}}=3040 \mathrm{~m} / \mathrm{s}$, la masse volumique est $\rho_{2}=2700 \mathrm{~kg} / \mathrm{m}^{3}$. La célérité de l'eau est choisie égale à $c_{1}=1493 \mathrm{~m} / \mathrm{s}$. Le fluide interne est de l'air $\left(c_{3}=330 \mathrm{~m} / \mathrm{s}, \rho_{3}=1,2 \mathrm{~kg} / \mathrm{m}^{3}\right)$. 


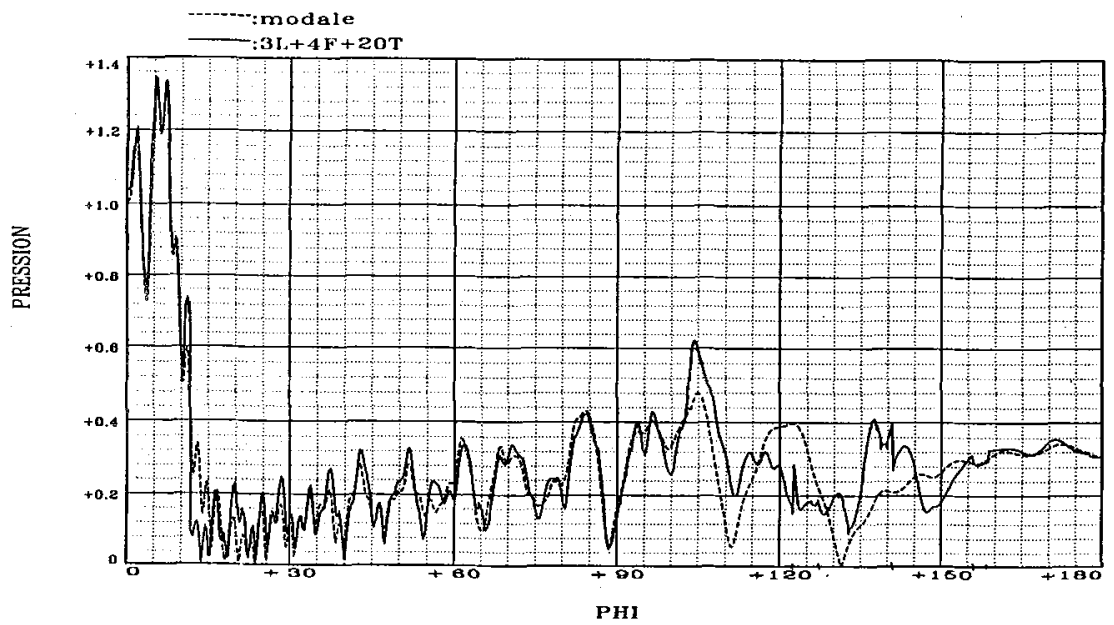

Fig. 3 : Amplitude de la pression diffusée par les rayons géométriques et les ondes de surface

\section{CONCLUSION}

Le présent travail constitue une application de la Théorie Géométrique de la Diffraction en Acoustique permettant de prédire le champ diffusé par un objet cylindrique immergé. Il s'agit d'une étape importante et indispensable pour modéliser des cibles de forme complexe.

\section{REMERCIEMENT}

Les auteurs désirent remercier A. DEREM et J.-M. CONOIR pour les nombreux échanges que leur ont permis de mener à bien ce travail.

\section{Bibliographie}

[1] A. DEREM, "La Diffusion Acoustique", chap. 9, N.GESPA, CEDOCAR, PARIS (1987), 189-279

[2] J.-L. ROUSSELOT, ACUSTICA, 79, (1993), (à paraître)

[3] G. QUENTIN, "La Diffusion Acoustique", chap.10, N.GESPA, CEDOCAR, PARIS (1987), 281-310

[4] J.-M. CONOIR, A. GERARD, A. DEREM, J. Acoust. 4 (1991), 159-200 